\title{
A simplified model of screening questionnaire and home monitoring for obstructive sleep apnoea in primary care
}

\author{
Ching Li Chai-Coetzer, ${ }^{1,2}$ Nick A Antic, ${ }^{1,2}$ L Sharn Rowland, ${ }^{1}$ Peter G Catcheside, ${ }^{1,2,3}$ \\ Adrian Esterman, ${ }^{4}$ Richard L Reed, ${ }^{5}$ Helena Williams, ${ }^{6}$ Sandra Dunn, ${ }^{7}$ \\ R Doug McEvoy ${ }^{1,2,3}$
}

- Additional data are published online only. To view these files please visit the journal online (http://thorax.bmj.com).

${ }^{1}$ Adelaide Institute for Sleep Health, Repatriation General Hospital, Adelaide, South Australia, Australia

${ }^{2}$ Department of Medicine, Flinders University, Adelaide South Australia, Australia ${ }^{3}$ Discipline of Physiology, School of Molecular and Biomedical Sciences, University of Adelaide, Adelaide, South Australia, Australia ${ }^{4}$ Division of Health Sciences, University of South Australia, Adelaide, South Australia, Australia

${ }^{5}$ Discipline of General Practice, Flinders Promotion, Prevention and Primary Health Care Cluster, School of Medicine, Flinders University, Adelaide, South Australia

${ }^{6}$ General Practice Network South, Adelaide, South Australia, Australia ${ }^{7}$ Graduate School for Health Practice, Charles Darwin University, Darwin, Northern Territory, Australia

\section{Correspondence to}

Ching Li Chai-Coetzer, Adelaide Institute for Sleep Health, Repatriation General Hospital, Daws Road, Daw Park,

Adelaide, South Australia 5041, Australia;

chingli.chai@health.sa.gov.au

Received 4 October 2010 Accepted 29 November 2010 Published Online First 20 January 2011

\section{ABSTRACT}

Background To address the growing burden of disease and long waiting lists for sleep services, a simplified twostage model was developed and validated for identifying obstructive sleep apnoea (OSA) in primary care using a screening questionnaire followed by home sleep monitoring.

Methods 157 patients aged 25-70 years attending their primary care physician for any reason at six primary care clinics in rural and metropolitan regions of South Australia participated. The first 79 patients formed the development group and the next 78 patients the validation group. A screening questionnaire was developed from factors identified from sleep surveys, demographic and anthropometric data to be predictive of moderate to severe OSA. Receiver operating characteristic (ROC) curve analysis was used to validate the two-channel ApneaLink device against full polysomnography. The diagnostic accuracy of the overall two-stage model was then evaluated.

Results Snoring, waist circumference, witnessed apnoeas and age were predictive of OSA and incorporated into a screening questionnaire $(\mathrm{ROC}$ area under curve (AUC) $0.84,95 \% \mathrm{Cl} 0.75$ to $0.94, \mathrm{p}<0.001$ ). ApneaLink oximetry with a $3 \%$ dip rate was highly predictive of OSA (AUC 0.96, 95\% Cl 0.91 to 1.0, $\mathrm{p}<0.001)$. The two-stage diagnostic model showed a sensitivity of 0.97 (95\% Cl 0.81 to 1.00$)$ and specificity of 0.87 (95\% $\mathrm{Cl} 0.74$ to 0.95 ) in the development group, and a sensitivity of $0.88(95 \% \mathrm{Cl} 0.60$ to 0.98$)$ and specificity of 0.82 ( $95 \% \mathrm{Cl} 0.70$ to 0.90$)$ in the validation group.

Conclusion A two-stage model of screening questionnaire followed by home oximetry can accurately identify patients with OSA in primary care and has the potential to expedite care for patients with this common sleep disorder.

\section{INTRODUCTION}

Obstructive sleep apnoea (OSA) affects $9-24 \%$ of middle-aged adults, with at least $4 \%$ of men and $2 \%$ of women suffering from symptomatic disease. ${ }^{1}$ These figures, however, probably underestimate the current prevalence of OSA given more recent population trends in obesity. ${ }^{2}{ }^{3}$ OSA is associated with an increased risk of hypertension, motor vehicle accidents, neurocognitive impairment and cardiovascular disease. ${ }^{4-7}$ Treatment of OSA can reduce the health-related consequences of disease ${ }^{78}$ and is highly cost-effective. ${ }^{9}$ Despite increasing awareness of its adverse health consequences, OSA remains significantly underdiagnosed. ${ }^{10}$

A major impediment to OSA service access is the reliance on diagnostic laboratory-based polysomnography (PSG) which is labour-intensive, costly and has limited availability. ${ }^{11}$ Another impediment is the relative dearth of qualified sleep physicians. Simplified strategies have been proposed to address these issues, including clinical prediction models ${ }^{12-16}$ and home-based strategies incorporating portable sleep monitoring and autotitrating continuous positive airway pressure (CPAP). ${ }^{17}$ A recent study showed that primary responsibility for the care of OSA can be assumed by sleep-trained nurses, and therefore potentially other health professionals, without compromising patient outcomes. ${ }^{18}$

Significant potential exists to broaden the scope of OSA diagnosis and management within the primary care setting. Almost one-third of primary care patients have a high likelihood of OSA, ${ }^{19}$ yet doctors often fail to ask about features of OSA and patients frequently fail to report sleep-related symptoms. ${ }^{20} \mathrm{~A}$ possible barrier is the absence of a simple validated screening tool suitable for a busy primary care environment. The ideal diagnostic screen would contain no more than five items and be quick to administer and interpret without the need for specialised equipment or examination techniques.

The Berlin questionnaire is the only OSA questionnaire developed for and validated in primary care $^{16}$ and categorises patients as either high or low risk for OSA based on self-reports of snoring, daytime sleepiness, hypertension and obesity. The 11 questions were chosen by a panel of sleep physicians without prior evaluation as to their respective discriminatory values. Although published a decade ago, the Berlin questionnaire is not widely used, possibly because of the time required for completion and scoring. Other screening tools have only been tested on selected populations in specialist sleep centres ${ }^{12-15}$ and, like the Berlin questionnaire, have complex scoring systems ${ }^{12-14}$ and/or require measurements of facial and oropharyngeal anatomy. ${ }^{15}$ Nevertheless, questionnaires alone may not provide a sufficient basis for diagnostic and treatment decisions in OSA. Consequently, suitably simple, accurate and validated strategies capturing both symptomatology 
and objective signs of overnight breathing disturbances are needed.

The aim of this study was to develop and validate a simplified two-stage method for identifying moderate to severe OSA in primary care consisting of an easy-to-administer screening questionnaire followed by home sleep apnoea monitoring.

\section{METHODS}

A detailed account of the methodology is available in the online supplement. The study protocol was approved by the institutional research ethics committees at the Repatriation General Hospital and Flinders Medical Centre, South Australia, and participants provided written informed consent. The study was designed to meet the STARD guidelines for reports of diagnostic accuracy. $^{21}$

\section{Survey distribution and patient selection}

Patients aged $25-70$ years attending six primary care clinics for any reason between June 2007 and April 2008 were asked to complete a general health questionnaire, Epworth Sleepiness Scale $(E S S)^{22}$ and Berlin questionnaire. Pregnant women and patients with significant cognitive impairment, a poorlycontrolled psychiatric disorder or who had previously received treatment for OSA were excluded.

Reasoning that the true prevalence of moderate to severe OSA in our study population would be relatively low, an 'OSAenriched' patient sample was selected for home sleep studies to minimise the CIs around estimates of diagnostic accuracy: four 'high-risk' patients to every one 'low-risk' patient (according to Berlin questionnaire results) underwent simultaneous home PSG and monitoring with a two-channel portable device. The first half of patients recruited formed the development group and the second half of patients formed the validation group.

\section{Home sleep studies}

Patients were visited at home by a trained sleep nurse who took anthropometric measurements and attached sleep recording devices. Full PSG was conducted using a Somte multichannel recorder (Compumedics, Melbourne, Australia). While home PSG is not considered the true gold standard for diagnosis of OSA, the Somte device has previously been validated against full PSG in the laboratory setting ${ }^{23}$ and, in a previous study of the Sleep Heart Health Study cohort, respiratory disturbance index measures from unattended home PSG were found to be comparable to laboratory-based PSG. ${ }^{24}$ A single experienced sleep technician performed manual scoring of all home PSGs according to internationally agreed criteria. ${ }^{25}$ An apnoea was defined as a cessation of nasal flow lasting $\geq 10 \mathrm{~s}$. A hypopnoea was defined as a $50 \%$ decrease in nasal flow (or in both thoracic and abdominal excursions) lasting $\geq 10 \mathrm{~s}$ or a discernable decrease leading to $a \geq 3 \%$ oxygen desaturation or an EEG arousal. Moderate to severe OSA was defined as an apnoeahypopnoea index (AHI) of $\geq 30 / h$. A detailed description of the Somte PSG device and scoring criteria are provided in the online supplement.

The ApneaLink device (ResMed, Sydney, Australia) is a portable two-channel monitor which records oxygen saturation and nasal flow. Data were automatically analysed using ApneaLink software Version 6.00 to derive: (1) an $\mathrm{AHI}_{20,50}$ from the airflow signal, with apnoea defined as a reduction in airflow to $<20 \%$ of baseline and hypopnoea as a reduction in airflow to $20-50 \%$ of baseline for $\geq 10 \mathrm{~s}$; and (2) an oxygen desaturation index with $\geq 3 \%$ oxygen desaturations ( $3 \% \mathrm{ODI}$ ). Patients who failed PSG and/or ApneaLink studies were asked to repeat simultaneous home monitoring.

\section{Data analysis and statistics}

Differences between baseline characteristics of patients in the development and validation groups were examined using independent sample Student t tests, Wilcoxon sum rank tests or $\chi^{2}$ tests where appropriate (Stata Version 11.0). Further details are provided in the online supplement.

Chi-square automatic interaction detection (CHAID) ${ }^{26}$ was used to identify variables predictive of an AHI $\geq 30 / h$ in the development group and thus of potential use in the screening questionnaire (CHAID for Windows Version 6, Statistical Innovations Inc, 1993). A detailed description of CHAID and the choice of an AHI cut-off of $30 / \mathrm{h}$ to define moderate to severe OSA is provided in the online supplement. Potential predictor variables included gender, age, individual ESS and Berlin questionnaire items, total ESS score, body mass index (BMI), waist circumference and neck circumference. Variables predictive of OSA were entered into a logistic regression analysis and a simple scoring algorithm was derived using weightings from regression coefficients. Receiver operating characteristic (ROC) curve analyses were performed to assess the accuracy of the screening questionnaire as well as the ApneaLink-derived $\mathrm{AHI}_{20,50}$ and $3 \%$ ODI against a PSG AHI $\geq 30 / h$ (Microsoft Office Excel 2003, Microsoft Corporation). The superior ApneaLink parameter was selected for inclusion in the second step of the diagnostic model. Sensitivity, specificity, positive predictive value (PPV), negative predictive value (NPV), positive and negative likelihood ratios and overall test accuracy were calculated for both the questionnaire and ApneaLink to determine optimal cut-off values to maximise diagnostic efficiency. The accuracy of the final twostage model for moderate to severe OSA was then evaluated in the validation group.

\section{RESULTS}

The patient recruitment pathway is outlined in figure 1. Of the 1251 questionnaire packs returned from the primary care clinics, 461 patients (188 at high risk and 273 at low risk for OSA according to the Berlin questionnaire) were eligible for selection for home sleep studies. One hundred and seventy-six patients underwent home monitoring, with 157 performed successfully (123 patients at high risk and 34 at low risk for OSA). Nineteen patients were excluded owing to failed sleep studies, mainly from inadequate recording times or equipment failure, and either failed their second attempt or declined a repeat study. The development group consisted of the first 79 patients, while the remaining 78 patients made up the validation group.

Patient characteristics for the development and validation groups are shown in table 1. While both groups comprised predominantly middle-aged and overweight to obese individuals with approximately equal numbers of men and women, the development group was slightly older and had higher BMI, neck, waist and hip circumference measurements. The proportion of patients with moderate to severe OSA was higher in the development group. There was no difference in Berlin questionnaire risk rating or ESS score.

\section{Development data set}

\section{Screening questionnaire development}

Four variables were significantly predictive of moderate to severe OSA: (1) Berlin Questionnaire item 4: 'Has your snoring ever bothered other people?'; (2) waist circumference (men $>102 \mathrm{~cm}$, 
Figure 1 Patient recruitment pathway. OSA, obstructive sleep apnoea; PSG, polysomnography.



women >88 cm); (3) age $\geq 50$ years; (4) Berlin questionnaire item 5: 'Has anyone noticed that you stop breathing during your sleep?'. The results of the logistic regression analysis are shown in table 2. A 4-item screening tool was created and named the 'OSA50' questionnaire (figure 2). Points are allocated to each question, with snoring and waist circumference having the highest score of 3 points each and age and witnessed apnoeas gaining 2 points each, to give a maximum total score of 10 points.

On ROC curve analysis, the OSA50 questionnaire was significantly predictive of moderate to severe OSA with an AUC of 0.84 (95\% CI 0.75 to $0.94, p<0.001$, figure 3 ). Using a cut-off score of $\geq 5 / 10$, the screening questionnaire had a sensitivity of $100 \%$ (95\% CI $86 \%$ to $100 \%$ ), NPV of $100 \%$ (95\% CI $73 \%$ to $100 \%$ ), specificity of $29 \%$ (95\% CI $17 \%$ to $44 \%$ ) and PPV of $48 \%$ (95\% CI $35 \%$ to $63 \%)$.

Table 1 Characteristics of patients in development and validation groups

\begin{tabular}{llll}
\hline & $\begin{array}{l}\text { Development group } \\
(\mathbf{n = 7 9 )}\end{array}$ & $\begin{array}{l}\text { Validation group } \\
(\mathbf{n = 7 8 )}\end{array}$ & $\mathbf{p}$ Value \\
\hline Age, years & $55(45-62)$ & $50(40-58)$ & 0.015 \\
Men, $\mathrm{n}(\%)$ & $42(53 \%)$ & $34(44 \%)$ & 0.230 \\
$\mathrm{BMI}, \mathrm{kg} / \mathrm{m}^{2}$ & $31.7(28.8-36.1)$ & $29.3(25.5-33.8)$ & 0.014 \\
Neck circumference, cm & $40.3 \pm 4.2$ & $38.6 \pm 4.1$ & 0.011 \\
Waist circumference, cm & $106.7 \pm 13.9$ & $101.3 \pm 15.8$ & 0.024 \\
Hip circumference, cm & $113(105.5-121)$ & $106.5(100-117)$ & 0.004 \\
ESS & $8(4-10)$ & $7(5-10)$ & 0.997 \\
High risk, n (\%) & $65(82 \%)$ & $61(78 \%)$ & 0.521 \\
Total AHI, /h & $20.9(13.1-41.3)$ & $16.5(9.6-28.2)$ & 0.018 \\
AHI $\geq 30 / \mathrm{h}, \mathrm{n}(\%)$ & $31(39 \%)$ & $16(21 \%)$ & 0.010
\end{tabular}

Data are presented as mean $\pm \mathrm{SD}$, median (IQR) or $\mathrm{n}(\%)$.

$\mathrm{AHI}$, apnoea-hypopnoea index; BMI, body mass index; ESS, Epworth sleepiness scale.
Validation of Apnealink monitor

Two patients with successful home PSGs included in the questionnaire development had failed ApneaLink studies and were excluded from further analysis, leaving 77 patients for validation of the ApneaLink monitor and analysis of the overall two-stage diagnostic model. ROC curves for the ApneaLink 3\%ODI and $\mathrm{AHI}_{20,50}$ against PSG in the development group are shown in figure 4 . Both the $3 \% \mathrm{ODI}$ and $\mathrm{AHI}_{20,50}$ were highly predictive of moderate to severe OSA with ROC AUC values of $0.96(95 \% \mathrm{CI}$ 0.91 to $1.00, \mathrm{p}<0.001)$ and $0.95(95 \%$ CI 0.89 to $1.0, \mathrm{p}<0.001)$, respectively. The $3 \%$ ODI was selected for use in the two-stage model because oximetry was technically more reliable than nasal airflow measurements. Sixteen (9\%) of the 176 initial home sleep studies failed due to an inadequate airflow signal compared with only five $(3 \%)$ with failed oximetry.

\section{Two-stage diagnostic model}

The diagnostic characteristics of the two-stage model are shown in tables $3 \mathrm{~A}$ and 4 . Thirty patients in the development group had an AHI of $\geq 30 / h$. Using cut-off values of $\geq 5 / 10$ for the OSA50 questionnaire and $\geq 16 / \mathrm{h}$ for the $3 \%$ ODI, the two-stage model was capable of identifying moderate to severe OSA with a high sensitivity and specificity and had an overall diagnostic accuracy (sum of the true positive and true negative rate) of $91 \%$.

Table 2 Logistic regression analysis: factors associated with an apnoea-hypopnoea index $\geq 30 / h$ as determined by $\chi^{2}$ automatic interaction detection analysis

\begin{tabular}{llllr}
\hline Factors & Regression coefficient & SE & p Value & OR \\
\hline Snoring & 2.51 & 1.11 & 0.02 & 12.3 \\
Waist circumference & 2.22 & 0.92 & 0.02 & 9.2 \\
Apnoeas & 1.84 & 0.72 & 0.01 & 6.3 \\
Age 50+ & 1.49 & 0.66 & 0.02 & 4.4 \\
\hline
\end{tabular}


Figure 2 OSA50 screening questionnaire.

* Waist circumference to be measured at the level of the umbilicus.

\section{Validation data set}

The two-stage model was prospectively applied to the validation sample (tables $3 \mathrm{~B}$ and 4 ). Sixteen patients $(21 \%)$ in the validation group were confirmed to have moderate to severe OSA. The performance of the OSA50 questionnaire in the validation group was similar to that in the development group, with a ROC AUC of $0.75(95 \%$ CI 0.59 to $0.90, p<0.001)$. Twenty of the 78 patients $(26 \%)$ in the validation sample would have been excluded from further testing on the basis of a negative OSA50 questionnaire, only one of whom was positive for moderate to severe OSA on PSG. The two-stage model correctly identified moderate to severe OSA with a sensitivity and specificity of $>80 \%$, a very high NPV and overall diagnostic accuracy of $83 \%$.

\section{DISCUSSION}

Our results demonstrate that a simplified diagnostic model consisting of a screening questionnaire followed by home oximetry can identify patients in primary care with moderate to severe OSA with an overall accuracy exceeding $80 \%$. We targeted moderate to severe OSA as more severely affected patients have the highest morbidity and also adhere and respond to treatment better than those with mild disease. ${ }^{27}$ Compared with the



Figure 3 Receiver operating characteristic curve showing the performance of the OSA50 screening questionnaire in discriminating patients with moderate to severe obstructive sleep apnoea (apnoeahypopnoea index $\geq 30 / h$ ) in the development group $(n=79)$. AUC, area under the curve. standard pathway of care involving specialist review and laboratory-based testing, our diagnostic model has the potential to offer a low-cost and easily accessible strategy which can be initiated in primary care.

Our clinical prediction questionnaire is only the second such questionnaire to be developed specifically for primary care, and is a significant advance on the Berlin questionnaire ${ }^{16}$ because of its brevity and simple 10-point score. Previously published questionnaires have been derived from sleep clinic populations ${ }^{12-15}$ and require complex calculations or reference to specialised tables for interpretation. We created the OSA50 questionnaire with the hope that primary care providers would find it ideal for routine clinical use.

The four factors most predictive of OSA were the waist circumference, snoring, witnessed apnoeas and age, with similar variables identified in other studies. Two previously published screening tools include the multivariable apnoea risk (MAP) index ${ }^{13}$ and the sleep apnoea clinical score (SACS), ${ }^{12}$ both derived from sleep clinic cohorts. The MAP index is calculated using self-reports of snoring, gasping, witnessed apnoeas, BMI, age and gender. ${ }^{13}$ The ROC AUC for an AHI $\geq 10 / \mathrm{h}$ was 0.79 , with a sensitivity and specificity of $88 \%$ and $55 \%$, respectively. Flemons et $a^{12}$ found the strongest clinical predictors to be neck circumference, hypertension, snoring and partner reports of

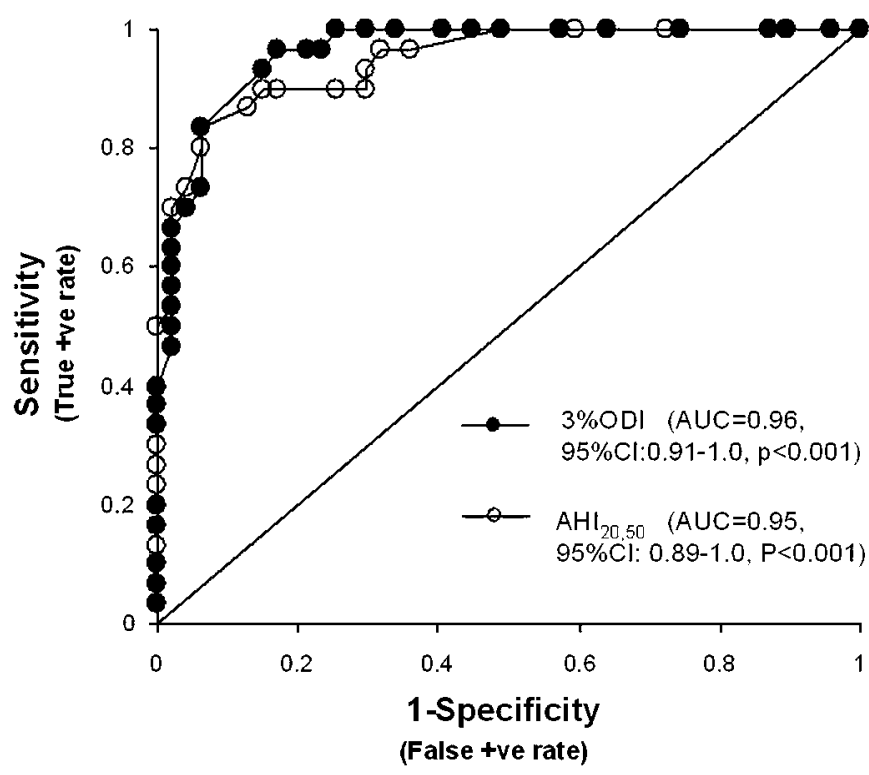

Figure 4 Receiver operating characteristic curves showing the performance of the ApneaLink $3 \% \mathrm{ODI}$ and $\mathrm{AHI}_{20,50}$ in diagnosing moderate to severe obstructive sleep apnoea $(A H I \geq 30 / h)$ in the development group. AHI, apnoea-hypopnoea index; ODI, oxygen desaturation index. 
Table 3 Contingency tables for development and validation groups: (A) Development group ( $n=77)$. (B) Validation group $(n=78)$

\section{A. Development Group $(n=77)$}

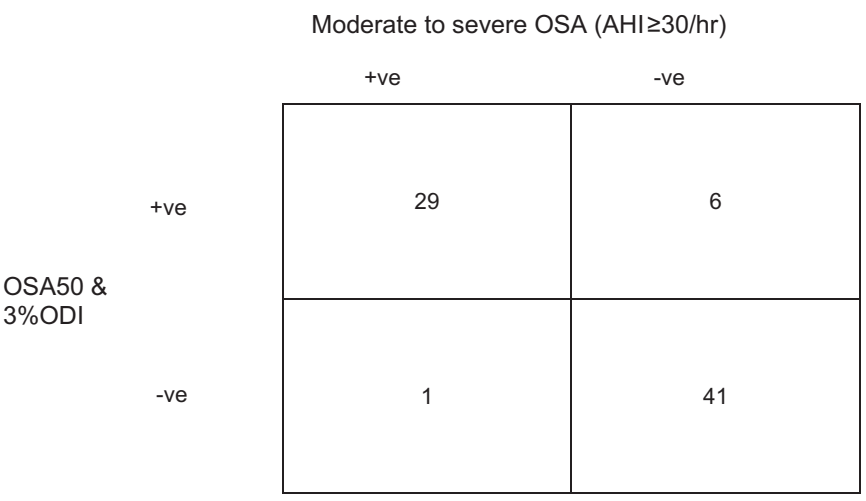

B. Validation Group $(n=78)$

Moderate to severe OSA (AHI $\geq 30 / h r)$

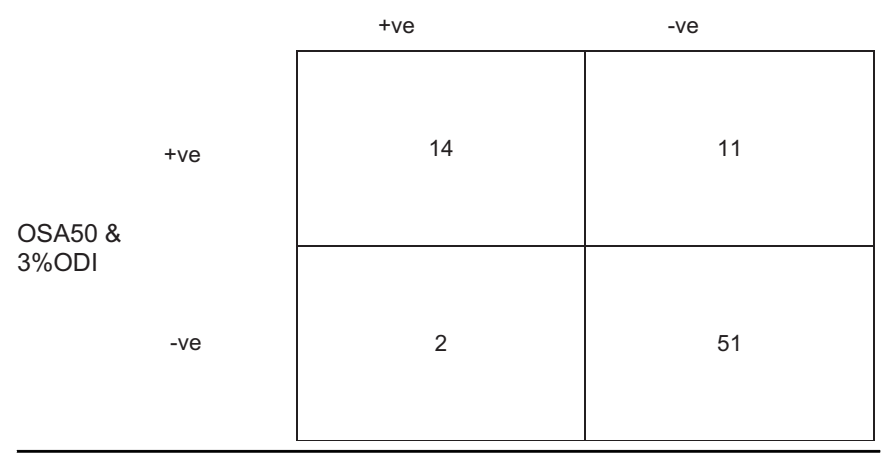

gasping or choking. For a SACS $>15$, the authors report a positive likelihood ratio of 5.2 and post-test probability of $81 \%$ for an AHI $>10 / h$. Other studies have incorporated craniofacial and oropharyngeal measurements into their prediction models, ${ }^{15}$ but it is unlikely that primary care providers would adopt a screening tool which required unfamiliar anatomical measurements not routinely used in clinical practice.

Although previously found to be an independent predictor of OSA, ${ }^{12}$ hypertension was not predictive of OSA in our study. Neck circumference has also been identified as a predictor of OSA. ${ }^{12}$ Interestingly, we found waist circumference to be a stronger predictor of OSA than other markers of obesity, with at least two other studies reporting similar findings. ${ }^{14} 28$ This is in keeping with the tracheal traction hypothesis for OSA, in which central obesity is believed to cause a reduction in lung

Table 4 Accuracy of two-stage diagnostic model (OSA50 score $\geq 5$ and ApneaLink 3\%ODI $\geq 16 / \mathrm{h}$ ) for identifying moderate to severe OSA

\begin{tabular}{|c|c|c|}
\hline & \multicolumn{2}{|c|}{ Moderate to severe OSA (AHI $\geq 30 / h$ ) } \\
\hline & Development group & Validation group \\
\hline Sensitivity & $0.97(0.81$ to 1.00$)$ & 0.88 (0.60 to 0.98$)$ \\
\hline Specificity & $0.87(0.74$ to 0.95$)$ & $0.82(0.70$ to 0.90$)$ \\
\hline PPV & $0.83(0.66$ to 0.93$)$ & $0.56(0.35$ to 0.75$)$ \\
\hline NPV & $0.98(0.86$ to 1.00$)$ & $0.96(0.86$ to 0.99$)$ \\
\hline $\mathrm{LR}+$ & 7.57 (3.58 to 16.03$)$ & $4.93(2.80$ to 8.70$)$ \\
\hline LR- & $0.04(0.01$ to 0.26$)$ & $0.15(0.04$ to 0.56$)$ \\
\hline
\end{tabular}

Data are presented as estimate $(95 \% \mathrm{Cl})$.

AHI, apnoea-hypopnoea index; LR, likelihood ratio; NPV, negative predictive value; ODI,

oxygen desaturation index; OSA, obstructive sleep apnoea; PPV, positive predictive value. volume during sleep leading to a loss of caudal traction on the upper airway thereby promoting pharyngeal collapse. ${ }^{29}$ As a questionnaire item, waist circumference is a simpler measure than BMI and is likely to be more familiar to patients than neck circumference. Patients should be aware of their pants size, of which a US men's size of '40 inches' and women's 'size 16' are equivalent to the questionnaire cut-off values of $>102 \mathrm{~cm}$ and $>88 \mathrm{~cm}$, respectively. Also, the National Cholesterol Education Program-Adult Treatment Panel (NCEP-ATP III) guidelines have included waist circumference in the diagnostic criteria for the metabolic syndrome using the same cut-off values. ${ }^{30}$ Primary care physicians are advised to promote intensive lifestyle modifications for patients with metabolic syndrome, ${ }^{31}$ which is associated with OSA and an independent risk factor for cardiovascular disease.

The diagnostic ability of four clinical prediction formulae, including the MAP index and SACS, was previously evaluated. ${ }^{32}$ The prediction models had high sensitivities of $76-96 \%$ with relatively low specificities of $13-54 \%$ and ROC AUC values of 0.67-0.74, similar to the results achieved by our OSA50 questionnaire. While the OSA50 questionnaire alone is not sufficiently accurate to discriminate between those with and without OSA, it is of value in confidently ruling out OSA and directing patients at high risk of disease for further diagnostic evaluation. To improve the accuracy of our diagnostic strategy, we have combined the OSA50 questionnaire with a second simple step of home sleep monitoring. We selected oximetry over nasal pressure for our two-stage model because the failure rate for the oximetry signal (3\%) was one-third that of the nasal pressure signal $(9 \%)$. We also felt that primary care physicians would be more familiar with the concept of oximetry measurement and have greater confidence in their ability to interpret study results. In contrast to laboratory-based PSG, overnight oximetry is less costly and can be self-administered in the patient's home without the need for technical staff. In 2007, the cost to Medicare (Australia's national health insurance system) for PSG was \$A485.65 per patient compared with \$A106.45 for overnight oximetry. In our validation sample the two-stage diagnostic model accurately identified patients with moderate to severe OSA with a sensitivity and specificity of $>80 \%$, with a negative result confidently ruling out disease (NPV 96\%). While the PPV appeared lower than ideal (56\%), review of the 11 patients classified as 'false positives' showed that all had evidence of at least mild OSA (minimum PSG AHI 18.9/h) and half reported excessive daytime sleepiness with ESS scores $\geq 12$ (see appendix 1 in online supplement), and therefore would be likely to obtain benefit from a trial of therapy.

Of note, neither the total ESS score nor individual items related to excessive sleepiness were predictive of OSA, similar to previous reports. ${ }^{12} 15$ Thus, while daytime sleepiness is a common complaint, it is not per se predictive of disordered breathing during sleep. Hypersomnolence, however, is an important determinant of whether CPAP should be recommended. Patients complaining of excessive sleepiness are more likely to respond to treatment and be compliant with CPAP than those with few symptoms. ${ }^{33} 34$ Our diagnostic model could be further refined by addition of a minimum ESS score cut-off, thereby specifically targeting hypersomnolent patients who would more likely benefit from urgent treatment.

A two-stage method for OSA using the MAP index and nocturnal oximetry was previously evaluated ${ }^{35}$ and, for an AHI $\geq 30 / \mathrm{h}$, had a sensitivity of $85 \%$, specificity of $97 \%$, PPV of $94 \%$ and NPV of $92 \%$. Mulgrew et al used a simplified diagnostic algorithm as part of an ambulatory management strategy for 
moderate to severe OSA which incorporated ESS, SACS and home oximetry. ${ }^{17}$ Of the 36 patients who scored positively on the diagnostic algorithm, 94\% (95\% CI $81 \%$ to $99 \%$ ) were correctly identified as having an AHI $>15 /$ h on subsequent PSG. These models, however, were evaluated in sleep disorder clinic populations that have a high pretest probability of disease. To our knowledge, our study is the first to evaluate a primary care population where a significant disease burden remains underrecognised.

We attempted to optimise the precision around our estimates by enriching samples with a higher proportion of 'high-risk' patients by the Berlin questionnaire, which may raise concerns as to whether our results can be generalised to a 'non-enriched' population. Theoretically, changes in disease prevalence do not affect the sensitivity or specificity of a test, but importantly influence predictive values of positive and negative test results. However, by enriching the study population with the Berlin questionnaire, it is possible that high-risk patients with OSA were more likely to score positive responses to selected items on the Berlin questionnaire than an unselected OSA patient sample. This could have influenced the final OSA50 screening questionnaire content and the sensitivity and specificity of the overall diagnostic process. A further effect of enriching a population with cases of moderate to severe OSA may be to falsely elevate the positive predictive value of the two-stage diagnostic screen. As it turned out, however, in our study employment of the 'enrichment' strategy had quite different effects in the development and validation samples. It markedly increased the prevalence of moderate to severe OSA in the development group but had little impact on the prevalence of moderate to severe OSA in the validation group since, in the validation group, 'highrisk' and 'low-risk' patients had similar rates of disease (21\% and $18 \%$, respectively). The reasons for this difference are not clear but may relate to the lower age and obesity rate in the validation group compared with the development group. We estimate, using back-extrapolation, that $20 \%$ of our original non-enriched primary care population would have had moderate to severe OSA, which is consistent with recent prevalence estimates from the general population. ${ }^{36}$ The estimated prevalence of moderate to severe OSA in the 'enriched' validation group was $21 \%$, which is virtually identical. We therefore believe that estimates of diagnostic accuracy from the validation sample are likely to be similar to that in a non-enriched primary care population. Furthermore, given similar overall rates of moderate to severe OSA in both the high-risk and low-risk groups in the validation sample and face validity of the final questionnaire items, the impact of the potential bias from using an enriched population would appear to be limited.

When applying sensitivity and specificity values obtained from our development and validation groups to a primary care population with a disease prevalence of $20 \%$, the PPV is in the range $55-65 \%$ and NPV in the range $97-99 \%$. The two-stage model therefore has its greatest value in ruling out disease. Patients considered 'false positive' are likely to have evidence of at least mild OSA (see above) and, if treatment decisions are based on symptoms such as sleepiness, then a trial of CPAP therapy is likely to be worthwhile with minimal risk of harm.

In summary, a two-stage diagnostic model consisting of a screening questionnaire followed by home oximetry can identify patients with clinically significant OSA in a primary care population with a high degree of accuracy. The model could potentially offer a cost-effective solution to the problem of currently overwhelmed laboratory-based sleep services and would be of particular benefit for rural and remote regions, as well as developing countries, where access to sleep services is limited. This simplified strategy, in conjunction with education of primary care physicians and provision of primary care-based management regimens, has the potential to significantly improve patient access to care and expedite treatment for this common sleep disorder.

Acknowledgements The authors would like to thank the Flinders Medical Centre Clinicians Trust for providing research scholarship support to CLC-C, ResMed for donation of ApneaLink monitors, Amanda McKenna for polysomnography scoring, Amanda Adams for her assistance with database entry and Allison Sulda for distribution of research questionnaires. The authors also wish to acknowledge the support of the following primary care clinics: Belair Medical Clinic, Chandlers Hill Surgery, Flagstaff Hill Medical Centre, Goolwa Medical Centre, Trinity Medical Centre Port Adelaide and Victor Medical Centre.

Funding This study was funded by the National Health and Medical Research Counci of Australia (grant 426744) who have had no role in the design, conduct, collection, management, analysis or interpretation of data, nor have they been involved in the preparation, review or approval of the manuscript for publication.

Competing interests RDMcE has been in receipt of two honoraria for speaking engagements from the sleep device company Philips-Respironics in the last 5 years. RDMcE and NAA have received a grant of $\$ 5 \mathrm{~m}$ from Philips-Respironics for a large randomised controlled trial of CPAP therapy for obstructive sleep apnoea with additional equipment donations from Philips-Respironics, ResMed and Compumedics (all sleep device companies) for this project. Equipment donations have also been received by RDMcE, CLC-C and NAA from Philips-Respironics, ResMed and Somnomed for a randomised controlled trial of simplified management of OSA in specialist and primary care settings. The source of direct funding for the latter two grants was the National Health and Medical Research Council of Australia. The remaining authors have no conflicts of interest.

Ethics approval This study was conducted with the approval of the Repatriation General Hospital and Flinders Medical Centre, South Australia.

Provenance and peer review Not commissioned; externally peer reviewed.

\section{REFERENCES}

1. Young T, Palta M, Dempsey J, et al. The occurrence of sleep-disordered breathing among middle-aged adults. N Eng/ J Med 1993;328:1230-5.

2. Ogden CL, Carroll MD, Curtin LR, et al. Prevalence of overweight and obesity in the United States, 1999-2004. JAMA 2006;295:1549-55

3. Peppard PE, Young T, Palta M, et al. Longitudinal study of moderate weight change and sleep-disordered breathing. JAMA 2000;284:3015-21.

4. Peppard PE, Young T, Palta M, et al. Prospective study of the association between sleep-disordered breathing and hypertension. N Engl J Med 2000;342:1378-84.

5. Teran-Santos J, Jimenez-Gomez A, Cordero-Guevara J. The association between sleep apnoea and the risk of traffic accidents. Cooperative Group Burgos-Santander. N Engl J Med 1999;340:847-51.

6. Engleman HM, Douglas NJ. Sleep 4: Sleepiness, cognitive function, and quality of life in obstructive sleep apnoea/hypopnoea syndrome. Thorax 2004;59:618-22.

7. Marin JM, Carrizo SJ, Vicente E, et al. Long-term cardiovascular outcomes in men with obstructive sleep apnoea-hypopnoea with or without treatment with continuous positive airway pressure: an observational study. Lancet 2005;365:1046-53.

8. George CF. Reduction in motor vehicle collisions following treatment of sleep apnoea with nasal CPAP. Thorax 2001;56:508-12.

9. Mar J, Rueda JR, Duran-Cantolla J, et al. The cost-effectiveness of nCPAP treatment in patients with moderate-to-severe obstructive sleep apnoea. Eur Respir $\mathrm{J}$ 2003;21:515-22.

10. Kapur V, Strohl KP, Redline $S$, et al. Underdiagnosis of sleep apnea syndrome in U.S communities. Sleep Breath 2002;6:49-54.

11. Flemons WW, Douglas NJ, Kuna ST, et al. Access to diagnosis and treatment of patients with suspected sleep apnea. Am J Respir Crit Care Med 2004;169:668-72.

12. Flemons WW, Whitelaw WA, Brant $\mathrm{R}$, et al. Likelihood ratios for a sleep apnea clinical prediction rule. Am J Respir Crit Care Med 1994;150:1279-85.

13. Maislin G, Pack Al, Kribbs NB, et al. A survey screen for prediction of apnea. Sleep 1995; 18:158-66

14. Deegan PC, McNicholas WT. Predictive value of clinical features for the obstructive sleep apnoea syndrome. Eur Respir J 1996;9:117-24.

15. Tsai WH, Remmers JE, Brant R, et al. A decision rule for diagnostic testing in obstructive sleep apnea. Am J Respir Crit Care Med 2003;167:1427-32.

16. Netzer NC, Stoohs RA, Netzer CM, et al. Using the Berlin questionnaire to identify patients at risk for the sleep apnea syndrome. Ann Intern Med 1999;131:485-91

17. Mulgrew AT, Fox N, Ayas NT, et al. Diagnosis and initial management of obstructive sleep apnea without polysomnography: a randomized validation study. Ann Intern Med 2007;146:157-66. 
18. Antic NA, Eckerman S, Esterman A, et al. A randomised controlled trial of nurse-led care for symptomatic moderate-severe obstructive sleep apnea. Am J Respir Crit Care Med 2009;179:501-8.

19. Netzer NC, Hoegel JJ, Loube D, et al. Prevalence of symptoms and risk of sleep apnea in primary care. Chest 2003;124:1406-14.

20. Rahaghi F, Basner RC. Delayed diagnosis of obstructive sleep apnea: don't ask, don't tell. Sleep Breath 1999;3:119-24.

21. Bossuyt PM, Reitsma JB, Bruns D, et al. Towards complete and accurate reporting of studies of diagnostic accuracy: the STARD initiative. Clin Chem 2003; 49:1-6.

22. Johns MW. A new method for measuring daytime sleepiness: the Epworth sleepiness scale. Sleep 1991;14:540-5.

23. Cunnington D, Himanshu G, Teichtahl H. Accuracy of an ambulatory device for the diagnosis of sleep disordered breathing. Indian J Sleep Med 2009:4:143-8

24. Iber $\mathbf{C}$, Redline S, Kaplin Gilpin AM, et al. Polysomnography performed in the unattended home versus the attended laboratory setting: Sleep Heart Health Study methodology. Sleep 2004;27:536-40.

25. American Academy of Sleep Medicine Task Force. Sleep-related breathing disorders in adults: recommendations for syndrome definition and measurement techniques in clinical research. Sleep 1999;22:667-89.

26. Kass GV. An exploratory technique for investigating large quantities of categorical data. App/ Stat 1980;29:119-27.
27. Barnes M, Houston D, Worsnop CJ, et al. A randomized controlled trial of continuous positive airway pressure in mild obstructive sleep apnea. Am J Respir Crit Care Med 2002;165:773-80.

28. Davidson TM, Patel MR. Waist circumference and sleep disordered breathing. Laryngoscope 2008;118:339-47.

29. Eckert D, Malhotra A. Pathophysiology of adult obstructive sleep apnea. Proc Am Thorac Soc 2008;5:144-53.

30. Expert Panel on Detection, Evaluation, and Treatment of High Blood Cholesterol in Adults. Executive Summary of the Third Report of the National Cholesterol Education Program (NCEP) Expert Panel on detection, evaluation, and treatment of high blood cholesterol in adults (Adult Treatment Panel III). JAMA 2001:285:2486-97.

31. Deen D. Metabolic syndrome: time for action. Am Fam Physician 2004;69:2875-82

32. Rowley JA, Aboussouan LS, Badr MS. The use of clinical prediction formulas in the evaluation of obstructive sleep apnea. Sleep 2000:23:929-38.

33. Wolkove N, Baltzan M, Kamel H, et al. Long-term compliance with continuous positive airway pressure in patients with obstructive sleep apnea. Can Respir J 2008;15:365-9.

34. Engleman HM, Asgari-Jirhandeh N, McLeod AL, et al. Self reported use of CPAP and benefits of CPAP therapy: a patient survey. Chest 1996;109:1470-6.

35. Gurubhagavatula I, Maislin G, Pack Al. An algorithm to stratify sleep apnea risk in a sleep disorders clinic population. Am J Respir Crit Care Med 2001;164:1904-9.

36. Tufik S, Santos-Silva R, Taddei JA, et al. Obstructive sleep apnea syndrome in the Sao Paulo Epidemiologic Sleep Study. Sleep Med 2010:11:441-6.

\section{Lung alert}

\section{Treatment with $\beta$-blockers may reduce exacerbations and mortality in patients with COPD}

Cardiovascular disease is a significant burden in patients with chronic obstructive pulmonary disease (COPD). Shortness of breath and fatigue may be misinterpreted as symptoms of COPD, leaving cardiovascular disease unrecognised. Beta-blockers improve survival in ischaemic heart disease and heart failure. Many physicians avoid prescribing $\beta$-blockers in COPD because of concerns regarding bronchoconstriction and 'competition' with $\beta$-agonists, despite meta-analyses showing that they are well tolerated in this group.

In this observational cohort study, data from 2230 patients coded as having chronic bronchitis or emphysema according to GP electronic medical records between 1995 and 2005 were analysed. All-cause mortality and exacerbations of COPD were measured as end points. The authors found that 665 patients $(29.8 \%)$ were prescribed $\beta$-blockers for a variety of conditions. A reduction in all-cause mortality was observed in the $\beta$-blocker group with an HR of 0.7. This effect was seen in cardioselective and non-selective $\beta$-blocker use. A reduction in exacerbations of COPD was also noted. These findings were also observed in patients with COPD without overt cardiovascular disease.

This study demonstrates that $\beta$-blockers may be beneficial in COPD, but a randomised controlled trial would be needed to confirm these findings. The study used a diagnosis of COPD based on clinical coding rather than spirometry which may have resulted in misclassification of some patients. The findings in this large sample with a long follow-up should alert physicians to consider cardiovascular disease when assessing all patients.

Rutten F, Zuithoff NPA, Hak E, et al. $\beta$-Blockers may reduce mortality and risk of exacerbations in patients with chronic obstructive pulmonary disease. Arch Intern Med 2010;170:880-7.

\section{Castle}

Correspondence to L Castle, Specialist Registrar, Department of Respiratory Medicine, Churchill Hospital, Oxford OX3 7LJ, UK; liannecastle@doctors.org.uk

Published Online First 13 October 2010

Thorax 2011;66:219. doi:10.1136/thx.2010.145698 